\title{
Cultura para tejer la ciudad fragmentada. Políticas culturales y la construcción de ciudades inclusivas
}

\author{
Emma Grün Lorío
}

Artículo

\begin{abstract}
Resumen
La vitalidad de la cultura es necesaria para la ciudad. Permea todas las esferas de la vida urbana y constituye la base de las libertades, intercambios de ideas y bienestar social (UNESCO, 2017). En este marco, el presente escrito trata el abordaje de las estrategias culturales como objeto de las políticas públicas y de intervenciones urbanas en programas de revitalización urbana para mitigar las consecuencias de la fragmentación urbana presente en las ciudades latinoamericanas la cual se caracteriza por una baja integración espacial y por tener una sociedad desvinculada. Se toma como punto de partida, el reconocimiento de los derechos culturales de toda la ciudadanía abordando pautas para subrayar la importancia de la cultura en el desarrollo sostenible de las ciudades, presentando entre las más destacadas: el rol de las autoridades locales en la traducción de las políticas culturales internacionales para la creación y apoyo de espacios de acción y diálogo y la planificación e implementación de políticas y programas culturales que reconozcan el valor del patrimonio tangible e intangible, la diversidad cultural y la creatividad; y el rol del diseño urbano inclusivo orientado a las personas, capaz de capitalizar el intercambio efectivo entre una ciudadanía diversa y de contrarrestar la violencia urbana, entre otros desafíos urbanos provenientes de las presiones de la urbanización masiva y acelerada. Así, este escrito pretende reflexionar sobre el desarrollo de mecanismos eficaces para la creación de políticas y estrategias urbanas responsables que se basen en la realidad de nuestras ciudades y promuevan enfoques inclusivos para la erradicación de todas las formas de discriminación en las ciudades.
\end{abstract}

Palabras clave: derechos culturales; desarrollo urbano; fragmentación urbana; inclusión urbana.

Culture to weave the fragmented city. Cultural policies and the construction of inclusive cities

\section{Abstract}

Cultural vitality is necessary for the City. It permeates all of the spheres of urban life and lays the basis for liberties, exchange of ideas and social welfare (UNESCO, 2017). In this context, this article will deal with cultural strategies as an object of public policies and urban interventions in urban revitalization programmes oriented to mitigate the consequences of urban fragmentation present in Latin American cities which is characterized by low spatial integration and a disassociated society. The starting point is the acknowledgment of cultural rights for all citizens, addressing guidelines which remark the importance of culture in the sustainable development of cities presenting amongst the outstanding: the role of local authorities in the translation of international cultural policies for the creation and support of action and dialogue spaces and the planning and implementation of policies and cultural programmes which recognize the value of intangible and tangible patrimony, cultural diversity, creativity; and the role of inclusive people-oriented urban design able to capitalize the effective exchange of diverse citizenship as well as counter urban violence among other urban challenges stemming from the pressures of rapid and massive urbanization. This article aims to reflect on the development of effective mechanisms for the creation of responsible urban policies and strategies that are based on the reality of our cities and promote inclusive approaches for the eradication of all forms of discrimination in cities.

Keywords: cultural rights; urban development; urban fragmentation; urban inclusion. 


\section{Introducción}

"Como hipótesis tentativa, se puede proponer que en la ciudad preindustrial, la distancia social era grande pero la distancia espacial era pequeña (por ejemplo, sociedades coloniales propietarias de esclavos); en la ciudad industrial, la distancia social se redujo (con la formación de la clase media) pero la distancia espacial mayor (con el aumento de la segregación socioespacial) y en la ciudad postindustrial fragmentada, la distancia social se hizo mayor (con una mayor desigualdad de ingresos ) y la distancia espacial se ha reducido. Sin embargo, la relación entre accesibilidad y proximidad se ha roto en condiciones de fragmentación urbana." (Burgess, 2005, p. 22)

n la actualidad, el modelo de urbanización de las ciudades latinoamericanas ha seguido lógicas que privilegian el abastecimiento de individuos y la diferenciación selectiva. Aunque es ampliamente reconocido que toda la población urbana tiene derecho a participar en la vida urbana, tanto espacial como social, la realidad en nuestras ciudades se encuentra fragmentada urbana y espacialmente. La baja integración espacial con una sociedad desvinculada, una red de infraestructura desequilibrada (como rutas del eje principal y acceso a altas concentraciones de transporte público), la distribución desigual de las instalaciones públicas (como áreas de recreación y centros formales de programas culturales) y una descontinuación del tejido urbano por el incremento de los archipiélagos urbanos (como la comunidad cerrada y los barrios marginales), constituyen, entre otros, los rasgos más prominentes.

El fenómeno urbano de la fragmentación espacial de nuestras ciudades latinoamericanas se da en un contexto de crecimiento acelerado y dinamismo migratorio de la población. Según cifras de ONU-Hábitat, actualmente en el mundo hay aproximadamente un millón de ciudades que albergan a 3.900 millones de personas, es decir, más de la mitad de la población mundial y para el año 2050 se estima que los habitantes urbanos aumentarán a dos tercios de los habitantes del mundo. Esta urbanización masiva y acelerada tiende a exacerbar algunos de los desafíos urbanos que identificamos hoy en nuestras ciudades, dando lugar a más barrios marginales y menos acceso a los espacios públicos, así como a un mayor impacto medioambiental negativo. Las consecuencias potenciales de este proceso son el desempleo, la desigualdad, la discriminación y la violencia.

Asimismo, la globalización está aumentando la movilidad de personas e ideas. Hoy en día, casi ningún país es culturalmente homogéneo. Los 5000 grupos étnicos del mundo se concentran en los casi 200 países del mundo; dos tercios de estos países tienen, por lo menos, una minoría sustancial: un grupo étnico o religioso que constituye al menos el $10 \%$ de la población (Programa de las Naciones Unidas para el Desarrollo, 2004). Y es en este contexto que existe un miedo a que la cultura pueda perder su autonomía y el contenido crítico que constituye su propia esencia. Allen Scott (2011), advierte que al estudiar los movimientos recientes de globalización éstos no sólo integran y generan mestizajes, sino que también segregan, producen nuevas desigualdades y estimulan reacciones diferencialistas. Es así como, la cuestión sobre la identidad cultural representa un desafío central de hoy y del futuro que ningún pueblo puede eludir.

Aún en este contexto, las ciudades aportan el $70 \%$ del PIB mundial y son motores de desarrollo económico, social y cultural. En las palabras de Irina Bokova, Directora General de la Organización de las Naciones Unidas para la Educación, la Ciencia y la Cultura (UNESCO), "las ciudades son laboratorios vivos donde se determina cómo afrontar, gestionar y experimentar algunos de los mayores desafíos a los que nos enfrentamos" (UNESCO, 2017, p. 5). En esa línea, este escrito respalda que la cultura ocupa un lugar central en la renovación y la innovación urbana, y que puede ser determinante para el éxito de las políticas de desarrollo sostenible que hagan frente a múltiples desafíos urbanos, como impulsor y facilitador del desarrollo y de las sociedades centradas en las personas (UNESCO, 2017). Particularmente, en ciudades donde "cada vez existen menos lugares de encuentro universal y el principio de exclusividad es el que con mayor potencia rige la producción de nuevas tipologías urbanas" (Kozak, 2018, p. 19), se debe de atender de manera proactiva la necesidad de desarrollar estrategias urbanas que impulsen la cultura y promuevan la diversidad de expresiones culturales 
"para promover la comprensión y tolerancia entre las personas, con el fin de facilitar la convivencia de unas poblaciones cada vez más multiculturales de las ciudades de hoy en día" (UNESCO, 2017, p. 133).

Es evidente que los desafíos de las poblaciones urbanas son diversos y que, en muchos casos, son críticos por la falta de satisfacción de necesidades básicas y la poca capacidad de las ciudades de satisfacer la demanda de infraestructura física y social. No obstante, se determina que la vida urbana debe atenderse paralelamente impulsando la cultura y la diversidad cultural en las ciudades. Lo antes mencionado cobra vital importancia ya que, así como lo define la Nueva Agenda Urbana, la cultura y la diversidad cultural son fuentes de enriquecimiento para la humanidad y realizan un aporte importante al desarrollo sostenible de las ciudades, los asentamientos humanos y la ciudadanía, empoderándola para que desempeñe una función activa y singular en las iniciativas de desarrollo.

Este escrito, en su estructura, muestra en la primera parte un marco de aproximación de la cultura como eje transversal para la construcción de ciudades inclusivas pasando por una interpretación de las aproximaciones a la definición del concepto de cultura, seguido de la relación entre ciudad y cultura, cerrando con una reflexión de su inclusión en planes y estrategias urbanas. En una segunda parte se aportan elementos claves para guiar la transformación de espacios públicos hacia escenarios culturales que promuevan la diversidad y la inclusión y así mitigar los efectos de la fragmentación espacial urbana. $Y$ en la tercera y última parte, se presentan las reflexiones finales del escrito, aportando pautas para la construcción de una visión a largo plazo y posibles abordajes para la creación de políticas culturales locales que permitan cumplir con las demandas del diseño y ejecución de proyectos urbanos.

\section{PARTE I: Cultura como eje transversal para la construcción de ciudades inclusivas}

\section{¿Qué entendemos por cultura? Interpretaciones de su carácter y función en la sociedad}

Aunque el concepto de cultura está sujeto a múltiples interpretaciones por la falta de anclas conceptuales claras, a continuación se presentan tres premisas que parten de la interpretación de tres acepciones básicas del concepto (Pascual, 2015): (1) aquella que concibe el término como "cultivo" de la mente, (2) la que pretende abarcar las formas de vida de una comunidad en todos sus aspectos, y (3) la que se restringe a las actividades vinculadas al patrimonio y las artes. Las aproximaciones que se presentan a continuación buscan describir el carácter y función de la cultura en la sociedad y, por lo tanto, dan pautas para reflexionar sobre el rol de las políticas culturales en el mundo.

La primera premisa reconoce a la cultura como un producto del sistema económico dominante. Esta interpretación parte de la acepción básica del concepto de cultura que concibe el término como "cultivo" de la mente. Uno de los precursores del tema, el doctor Teixeira Coelho en su publicación Diccionario crítico de política cultural: cultura e imaginario, percibe al hecho cultural como apéndice indisociable del sistema productivo, como se cita en Vargas (2003):

... la cultura es hoy claramente un producto al que se llega mediante un conjunto de operaciones bien definidas por el sistema de producción económico general. Es lo que sucede con el cine y también con la literatura y la música o, en una escala menor pero no diversa, con las artes plásticas y el teatro. Una película y un libro pueden tener un valor propio y ser signos notables de un grupo; no por eso dejan de ser productos económicos oriundos de un sistema de producción materialmente bien definido.

Así, esta premisa se sustenta en el vínculo manifiesto entre economía y cultura; como lo confirma Scott, "los grandes segmentos de la producción de la economía moderna están inscritos con un contenido cultural significativo, mientras que la cultura misma se suministra cada vez más en forma de bienes y servicios mercantilizados" (2001, p. 11). En los últimos años, los científicos sociales de muchos campos diferentes han 
señalado que lo económico está irremediablemente integrado en lo cultural, y varios geógrafos han respondido a esta idea pidiendo una geografía cultural vigorosa de las prácticas económicas (Scott, 2001).

Además, la cultura es una actividad que genera importantes economías externas como potenciador del capital humano, el empleo, catalizador de otras economías y el turismo (Asociación de Gestores y Técnicos Culturales). Sin embargo, si la cultura solo justifica su existencia a través de la rentabilidad económica, buena parte de las expresiones culturales estarán condenadas a desaparecer por no ser rentables o sostenibles, por no tener demanda o no aportar saberes útiles para la búsqueda de un empleo (Díez, 2015).

Esto trae a colación la segunda premisa: la cultura no es lineal ni estática. Esta premisa se basa en la comprensión de que todas las culturas contemporáneas son híbridas; entendiendo como hibridación los procesos socioculturales en los que estructuras o prácticas discretas, que existían en forma separada, se combinan para generar nuevas estructuras, objetos y prácticas (García, 2012). Es así como, a lo largo del tiempo, se ha llevado un proceso de integración incentivado por los encuentros, la interacción y la reconstrucción de diferentes culturas locales, así como lo explica Edward Said (Said cit. en Pascual, 2015):

... las civilizaciones no son compartimentos estancos, ya que desde tiempos inmemoriales están en contacto entre sí y se contaminan mutuamente; en lo interno, desde un punto de vista étnico, cultural, social y religioso, las civilizaciones son también espacios plurales.

Esta premisa cobra importancia debido a que, una vez que existe el reconocimiento de la hibridación de las culturas, se reivindica y fortalece la condición de cualquier persona a elegir libremente y participar del moldeado de su cultura. Esta condición se denomina libertad cultural y parte del entendimiento que la cultura no es un conjunto estático de valores y prácticas, sino que, más bien, consiste en ampliar las opciones individuales y no en preservar valores ni prácticas como un fin en sí con una lealtad ciega hacia las tradiciones (PNUD, 2004).

En este contexto, se presenta la tercera premisa: la cultura es la dignidad de un pueblo. Esta premisa, al igual que la anterior, se basa en que la cultura tiene una estrecha asociación con los derechos humanos. Se parte del entendimiento de la cultura como la sumatoria de conocimientos, ideas, tradiciones y costumbres, y de que cada cultura busca una sensación de identidad y continuidad. Con esto dicho, la búsqueda se concentra en lograr el equilibrio entre el derecho a conocer y a que se respete su propia cultura y el derecho de conocer también las culturas que, en su diversidad, constituyen el patrimonio común de la humanidad (Declaración de Friburgo, 2007).

Particularmente, vale la pena hacer énfasis en el caso de los pueblos indígenas, quienes antes del 2007 (año en el que se adoptó la Declaración sobre los Derechos de los Pueblos Indígenas de Naciones Unidas) eran sujeto de asimilación forzosa y a la destrucción de su cultura cometiéndose atrocidades de todo tipo. Sin embargo, hoy en día, aunque falta mucho para asegurar a todos paz y seguridad, tienen establecidos varios derechos, entre ellos: el derecho a mantener y fortalecer sus instituciones culturales, el derecho a practicar y revitalizar sus tradiciones y costumbres culturales, el derecho a establecer y controlar sus instituciones y sistemas educativos brindando educación en sus propios idiomas, el derecho a la dignidad y a la diversidad de sus culturas, tradiciones, historias y aspiraciones, entre otros (Declaración sobre los Derechos de los Pueblos Indígenas de Naciones Unidas, 2007).

\section{Relación entre cultura y su escenario principal: la ciudad}

Por definición, las ciudades representan densas aglomeraciones de la vida social. Las ciudades son, por lo tanto, localidades marcadas por intrincadas redes de relaciones e intercambios humanos, lo que a su vez conduce a su tendencia a generar multiplicidad, flujo y eventos o experiencias inesperadas (Scott, 2001).

Bien señalan Buendía y Pino (2011) que si se miran las potencialidades de una ciudad a partir de lo que ésta ofrece y lo que puede ofrecer, los intercambios al interior de ella, los consumos que se gestan, la capacidad e infraestructura montada en torno 
Figura 1. Mural Mira del artista portugués Daniel > Eime. Largo de Artur Arcos, Oporto, Portugal. Fotografía: Ricardo Chaves Hernández. al ocio, el entretenimiento, las artes y la educación, nos damos cuenta de que todo está atravesado por una matriz cultural que impregna su huella en la ciudad. "Ésta, entonces, es más que una sumatoria de expresiones culturales y sociales; por ende, es cultura por sí misma" (Buendía y Pino, 2011, p. 23).

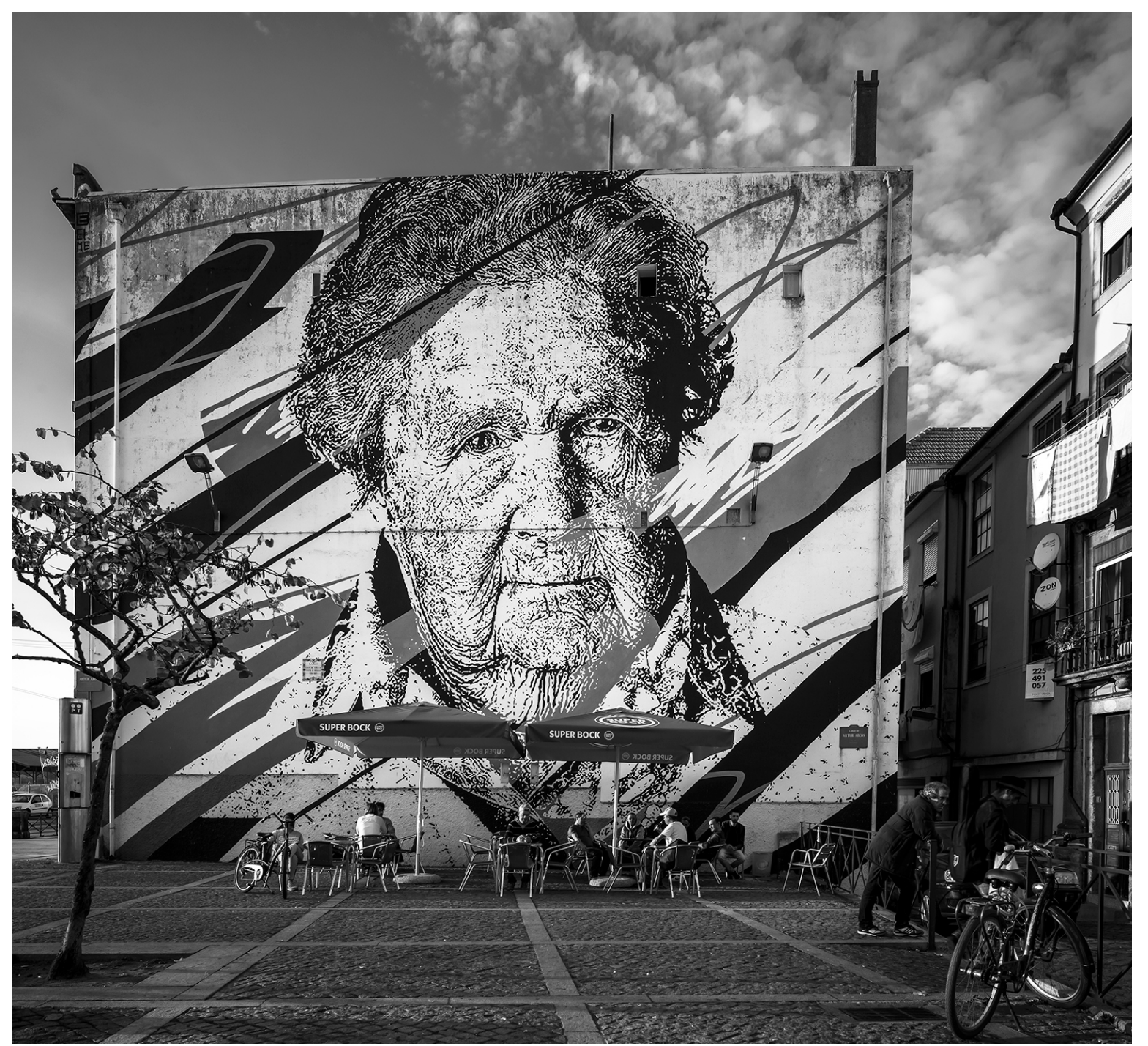

Hoy en día la diversidad cultural es un hecho innegable, podríamos decir que ésta es una de las características del mundo global de hoy. Las múltiples identidades particulares que luchan por el espacio dentro de la ciudad llevan a la creación de "otras ciudades" en su interior - algunas se dan por etnia, por género, otras por edad o procedencia geográfica -, en donde se dan cruces y fracturas sociales - "contactos" de unas identidades con otras - que advierten que nada en la ciudad es sólido, monolítico o estable (Buendía y Pino, 2011).

Se destaca el señalamiento de Borja y Castells (1997) sobre el hecho de que las sociedades son cada vez más diversas, y son las ciudades quienes más concentran tal diversidad. En esta realidad, lo clave es aprender a convivir con esta situación y sacarle el mejor provecho en beneficio de todos. Asimismo, estos autores destacaron que es necesario saber cómo se debe gestionar el intercambio cultural para que se dé en condiciones de equidad y que las diferencias étnicas sean respetadas y valoradas a fin de atenuar las desigualdades que han surgido debido a la discriminación (Borja y Castells, 1997).

\section{La cultura como componente prioritario de planes y estrategias urbanas}

Aun reconociendo que la vitalidad de la cultura es necesaria para la ciudad, es común que en planes y programas nacionales de cultura se encuentren formulaciones idénticas 0 muy parecidas a las que aparecen en los discursos y los lineamientos de los organismos internacionales (tales como la ONU, la UNESCO y la OEI) y que, por consiguiente, más que como normas, los derechos culturales se integran al diseño de políticas culturales bajo la modalidad de "intenciones", "condiciones para el desarrollo cultural" o principios que se derivan de tratados, acuerdos o declaraciones más recientes que tienen que ver con el respeto a los pueblos indígenas, las mujeres, los niños y la diversidad cultural 
Así como es el caso para la creación de todas las políticas públicas, en la planificación y el diseño de nuestras ciudades, en la creación de políticas culturales no hay una sola fórmula, cada situación es única. No obstante, así como lo expresa claramente Sakiko Fukuda-Parr, "la política cultural es el único enfoque posible, porque la alternativa es la supresión de la cultura" (Agencia Española de Cooperación Internacional, 2004, p. 29) $y$, dentro de este enfoque, la única solución sostenible es aceptar la diversidad.

Esto no requiere simplemente tolerancia individual, sino una política de estado que reconozca las distintas identidades culturales, un enfoque que puede ser ampliamente definido como «multiculturalismo». La democracia en estados multiétnicos necesita el reconocimiento constitucional y políticas pluralistas sobre idioma, representación política proporcional, religión y fiestas nacionales, así como combatir la profunda discriminación existente contra las minorías. Los mercados globales necesitan reconocer y apoyar el valor de la diversidad cultural y fomentar que las ideas, mercancías y personas fluyan, pero también debe enfrentarse a las asimetrías que amenazan a las culturas nacionales. Por ejemplo, la fuerza del mercado global significa que Hollywood puede destruir las industrias cinematográficas antaño prósperas de Italia y México. (Agencia Española de Cooperación Internacional, 2004, p. 29)

\title{
PARTE II: Espacios públicos como el principal escenario cultural
}

\begin{abstract}
El espacio público es un mecanismo fundamental para la socialización de la vida urbana. La negación de la ciudad es precisamente el aislamiento, la exclusión de la vida colectiva, la segregación. (...) En los espacios públicos se expresa la diversidad se produce el intercambio y se aprende la tolerancia. La calidad, la multiplicación y la accesibilidad de los espacios públicos definirán en gran medida la ciudadanía. (Borja y
\end{abstract}

Muxí, 2003, p. 67)

\section{La ciudad fragmentada y el espacio público}

En el contexto actual en el cual Castells define a las ciudades como "constelaciones discontinuas de fragmentos espaciales" (1999, p. 438), Carrión (2016) advierte que alrededor de los espacios públicos hay una especie de "agorafobia", asedio, rechazo 0 desprecio, al extremo de que la población los considera peligrosos y les teme porque no protegen ni son protegidos. Es un territorio abandonado incluso de la disputa social, su mantenimiento prácticamente no existe o es escaso: se ha convertido en guarida y no en hábitat (Borja y Muxí, 2003). Carrión (2016) continúa aportando que, resultado de este asedio al espacio público, la ciudad pierde las posibilidades de construcción y de cohesión sociales, se reduce la participación, se restringe la ciudadanía y se ausenta el estímulo a las prácticas de tolerancia. De ahí que los espacios públicos en el nuevo urbanismo de América Latina estén en peligro debido a diversos factores, entre ellos la fragmentación espacial.

La fragmentación lleva al habitante de la ciudad a ser visto como extranjero, porque cuando no camina por los senderos habituales hacia el lugar de trabajo o de residencia y sale de su territorialidad (barrio), se le exige identificación, como si fuera necesario un pasaporte para ir de un barrio hacia otro. Ahora nuestras ciudades no son de ciudadanos sino de forasteros. En otras palabras, la fragmentación ha dado lugar a la foraneidad en la ciudad, así como a la pérdida de los espacios referenciales para la construcción social (espacio público) y la pérdida del sentido de pertenencia. (Carrión, 2010, p. 195)

No obstante, esta tendencia se puede revertir. Según los estudios de Gehl (2014), si se ofrecen mejores espacios públicos, el uso se incrementará, un razonamiento válido tanto para los grandes espacios como para los más pequeños, llegando inclusive hasta la escala del banco y la silla. "El planeamiento puede influir los patrones de comportamiento y de uso de regiones y ciudades. Que la gente se sienta inclinada a caminar y a permanecer en los espacios urbanos es una cuestión íntimamente ligada a cómo se maneja la dimensión humana si se ofrecen los incentivos adecuados." (Gehl, 2014, p. 17). 
Figura 2. Escultura Forever bicycles del artista chino Ai Weiwei. Saint Mary Axe, Londres EC3A 8AG, Reino Unido.

Fotografía: Ricardo Chaves Hernández.

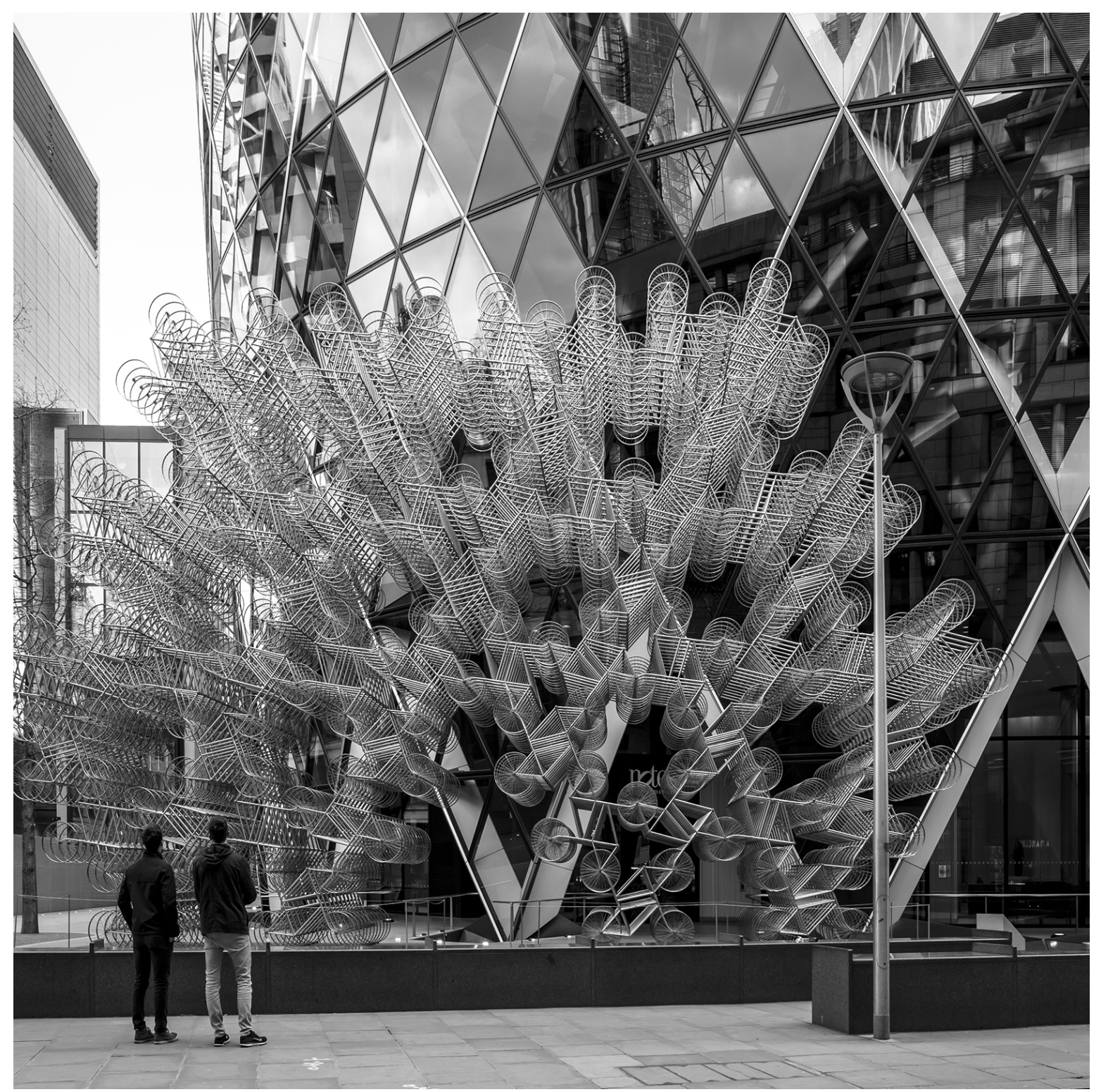

Pautas para espacios públicos inclusivos

Según cifras del Banco Mundial (2019), en el año 2050 la población urbana se duplicará y casi 7 de cada 10 personas vivirán en ciudades. Este punto de partida es importante porque, así como lo define Carrión (2016), "si la ciudad es el espacio que concentra la heterogeneidad social de un grupo poblacional grande y denso, se requieren espacios de encuentro y de contacto, tangibles (plazas) o intangibles (imaginarios), que permitan a los diversos reconstruir la unidad en la diversidad (la ciudad) y definir la ciudadanía (democracia). Esos lugares son justamente los espacios públicos" (p. 22).

Portal define que el deterioro de la calidad del espacio público impacta directamente en la cualidad de la ciudadanía y en las condiciones de su reproducción, debido a que "las relaciones sociales que se despliegan en esos espacios públicos son redefinidas constantemente no sólo en función de la capacidad organizativa y de apropiación que tienen los actores sociales que en él intervienen, sino también a partir de su calidad, es decir, de las condiciones materiales que los sustentan" (2016, p. 369).

En correspondencia con lo que aporta Burden (2014), que el diseño exitoso de un espacio público siempre depende de la experiencia personal y tomando como punto de partida el hecho que el diseño urbano inclusivo, orientado a las personas, es capaz de capitalizar el intercambio efectivo entre una ciudadanía diversa, se aportan tres pautas de diseño que deben de promoverse a la hora de formular proyectos para la creación o transformación de espacios públicos: (i) promover el intercambio diverso e inclusivo; (ii) ser un catalizador que permita la expresión de culturas diversas; y (iii) proporcionar espacios que se adaptan a un uso variado o dinámico de la vida urbana.

El espacio público es el lugar del intercambio por excelencia. Borja y Muxí, aportan que "si la diversidad y el intercambio son dimensiones fundamentales, la "ciudad ciudad" es aquella que optimiza las oportunidades de contacto, la que apuesta por la diferenciación y la mixtura funcional y social, la que multiplica los espacios de encuentro" (2003, p. 16). Por ende, promover el intercambio diverso e inclusivo debe ser un objetivo a la hora de diseñar, rehabilitar o revitalizar los espacios públicos. 
Figura 3. Proyecto de instalación escultórica pública por la Oficina de Arquitectura FAHR 021.3. Largo Amor de Perdição, Oporto, Portugal. Fotografía: Ricardo Chaves Hernández.

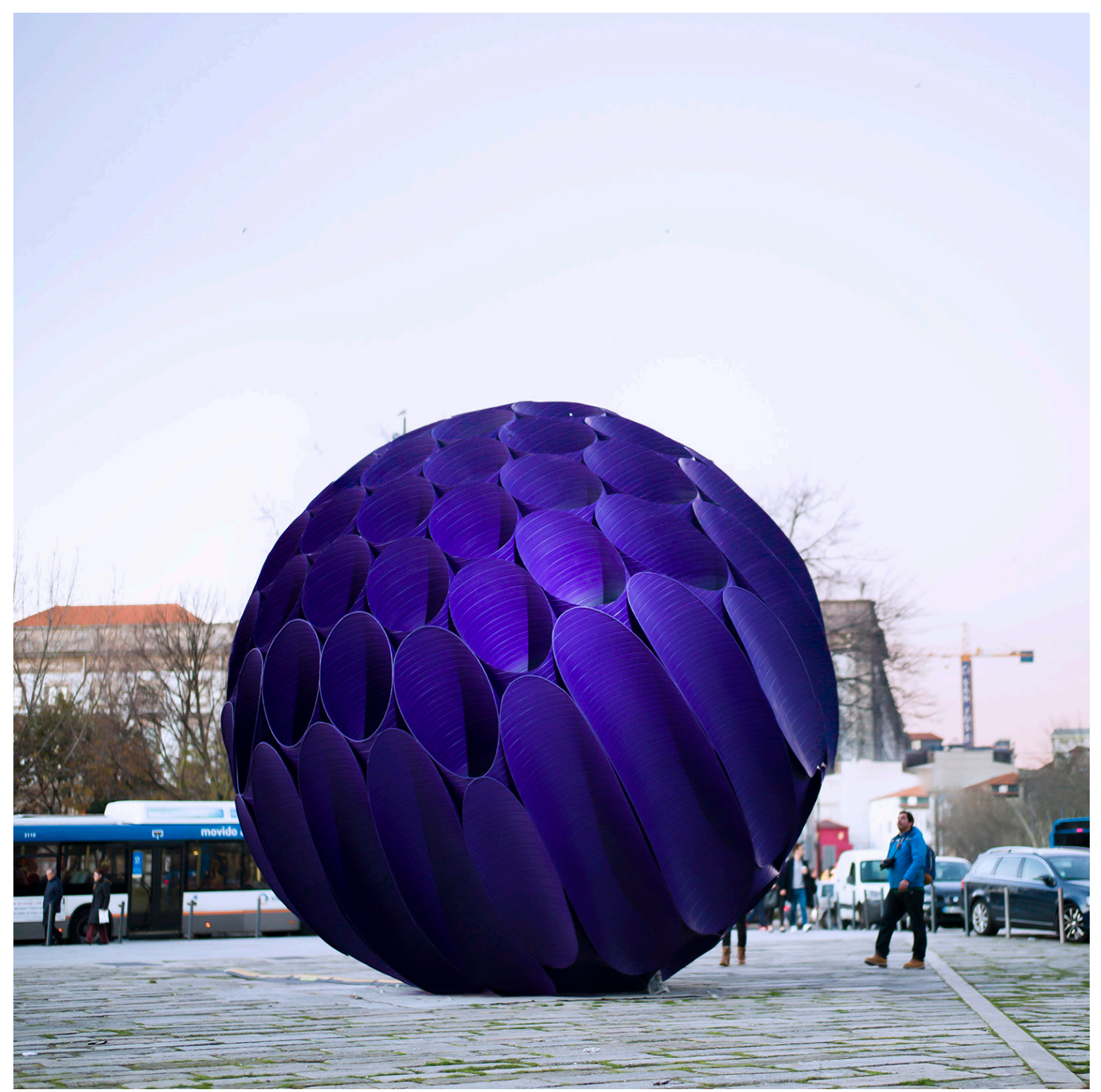

En este contexto, García-Domenech, contribuye que uno de los errores de diseño de espacios públicos ha sido la tendencia a racionalizar su uso, zonificándolo para albergar actividades concretas y especializadas (2014, p. 304). Actualmente, se promueve el carácter polivalente del espacio público para incentivar el uso y la estancia, "de manera que la versatilidad y flexibilidad en la zonificación se ha perfilado cada vez más como un índice de calidad en el diseño del espacio público urbano contemporáneo" (GarcíaDomenech, 2014, p. 305).

En las intervenciones artísticas, espontáneas e informales, sobre todo, se encuentra una oportunidad para promover la versatilidad del espacio urbano. Seijo (2014) aporta que para el arte contemporáneo el ejercicio plástico ya no se circunscribe necesariamente a las paredes de una galería o un museo, lo cual ha incrementado la cantidad de artistas, arquitectos y colectivos que desarrollan propuestas artísticas en el espacio urbano. Ciudades que han promovido el uso del espacio urbano como escenario artístico y cultural han reconocido un aporte al aumento de la calidad de los espacios públicos, esto se debe a que, así como define Lahbil Tagemouati (UNESCO, 2017, p. 185):

El arte y la cultura proporcionan las características necesarias para generar lugares positivos y destacables. Introducir el arte y la cultura en los procesos de planificación incrementa la participación pública, estimula el diseño colaborativo y añade valor a los proyectos de desarrollo. (...) La creación de espacios para la experiencia sensorial (vista, oído, olfato, tacto y gusto) debe ser impulsada por el arte y la cultura, los cuales refuerzan de manera colectiva y eficaz el sentido de pertenencia.

Consecuentemente, la diversidad cultural se puede expresar mediante la adaptabilidad de los espacios públicos a usos múltiples que se ajustan tanto a grupos culturales dominantes como diversos. Esta condición abona al importante objetivo de ser un catalizador que permita la expresión de culturas diversas, urgente, sobre todo porque como lo resume la Constitución de UNESCO: "(...) en el curso de la historia, la incomprensión mutua de los pueblos ha sido motivo de desconfianza y recelo entre las naciones, y causa de que sus desacuerdos hayan degenerado en guerra con harta frecuencia" (UNESCO, 2020, p. 7).

Este desafío socio-cultural recalca la demanda de dar calidad al espacio público y, así como definen Borja y Muxí (2003), buscar que cumpla con una función integradora 
compleja, combinando una función universalizadora con una función comunitaria o de grupo. Si el espacio público asegura una vida pública vibrante, siguiendo el argumento de Jacobs (1961), eventualmente reduciría el miedo y la discriminación. Wessel, a través de revisiones contemporáneas, coincide con la celebración de la diversidad urbana de Jacobs, sugiriendo que mayores niveles de contacto intergrupal generalmente corresponden con mayores niveles de tolerancia (2009, p. 11). Además, se fortalece la sugerencia de que la tolerancia se desarrolla en el contexto del espacio público y se reconoce que el intercambio entre culturas se basa en la socialización, entendida como "un proceso dialéctico que requiere tanto las relaciones entre todos y en todas direcciones como la integración en grupos de referencia de edad, de cultura, de clase" (Borja y Muxí, 2003, p. 66).

La clave para impulsar el intercambio en el espacio público radica en recurrir, así como aporta Burden (2014), a las habilidades "no como planificador urbano, sino como ciudadano" y el ciudadano es un usuario abstracto, complejo y cambiante, así como ya lo definía Norberg-Shulz (1975). Por ello, proporcionar espacios que se adaptan a un uso variado o dinámico de la vida urbana se torna en un objetivo vital en un marco donde "(...) la ciudadanía no es siempre la misma, es dinámica, puede cambiar rápidamente, sobre todo en periodos de movilizaciones sociales. No existe una ciudadanía para siempre, es una co-construcción histórica permanente, participativa, compleja, no lineal, democrática y popular" (Mejías y Henríquez, 2012, p. 202).

Existirán varios acercamientos para asegurar la vivacidad de los espacios públicos, pero cabe resaltar lo que plantea García-Domenech en una propuesta que combina la percepción estética con la social: "acumulación de elementos de interés, actividades y focos de atracción en los bordes de una plaza, genera una deliberada tensión entre los mismos que determina la vivacidad de uso del espacio público. (...) Para garantizar esa tensión urbana, resulta muy recomendable que tanto las actividades de atracción en los bordes participen del espacio público como que el propio espacio público participe de las actividades de atracción: son espacios de transición entre lo estrictamente público - espacio central de la plaza - y lo estrictamente privado - arquitecturas perimetrales -" (2014, p. 312).

Figura 4. Escultura Angel's Wing de Thomas Heatherwick. Paternoster Square, Londres. Fotografía: Ricardo Chaves Hernández.

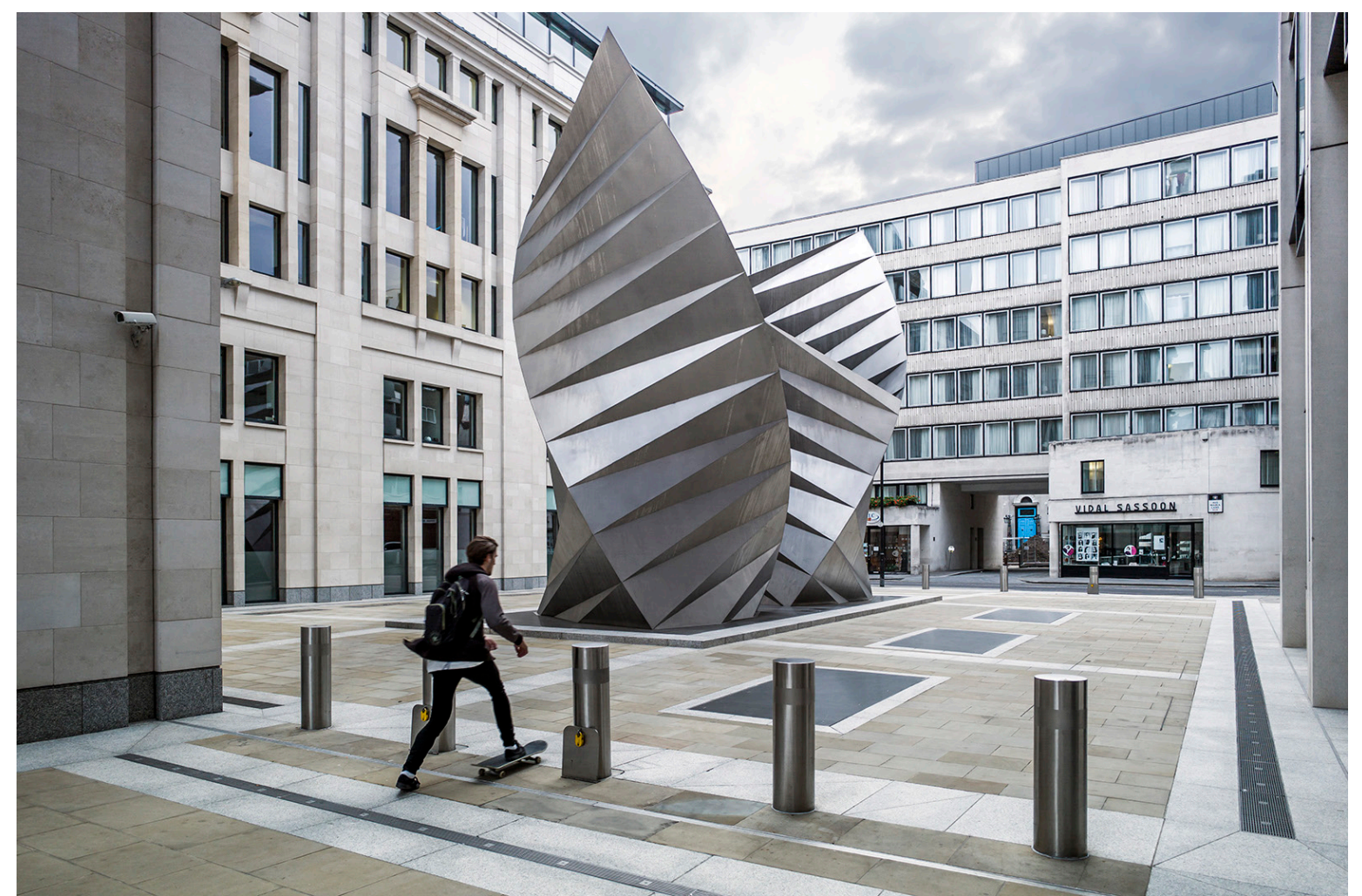

Además de usar los bordes, el diseño en sí de las plazas y las calles puede proveer experiencias visuales y artísticas, fungiendo como una plataforma de enlace donde el arte y las personas pueden encontrarse, para que sean atractivas y alienten la permanencia, y por lo tanto, el intercambio. Así como aporta Gehl, "en estos casos, el diseño y los detalles del espacio juegan un rol esencial, el cual puede ser reforzado apelando a los otros sentidos: una caída de agua, neblina, vapor, impresiones acústicas y aromáticas, por nombrar solo algunas" (2014, p. 178). En conclusión, siguiendo a Gehl (2014) los espacios públicos de calidad son espacios vivibles y calles vibrantes que deben ser abordados como áreas multifuncionales que sirvan para la interacción social, el intercambio económico y la expresión cultural para una amplia variedad de participantes. 
Figura 5. Obra artística History Trees de Ackroyd > y Harvey. Queen Elizabeth Olympic Park, Londres.

Fotografía: Ricardo Chaves Hernández.

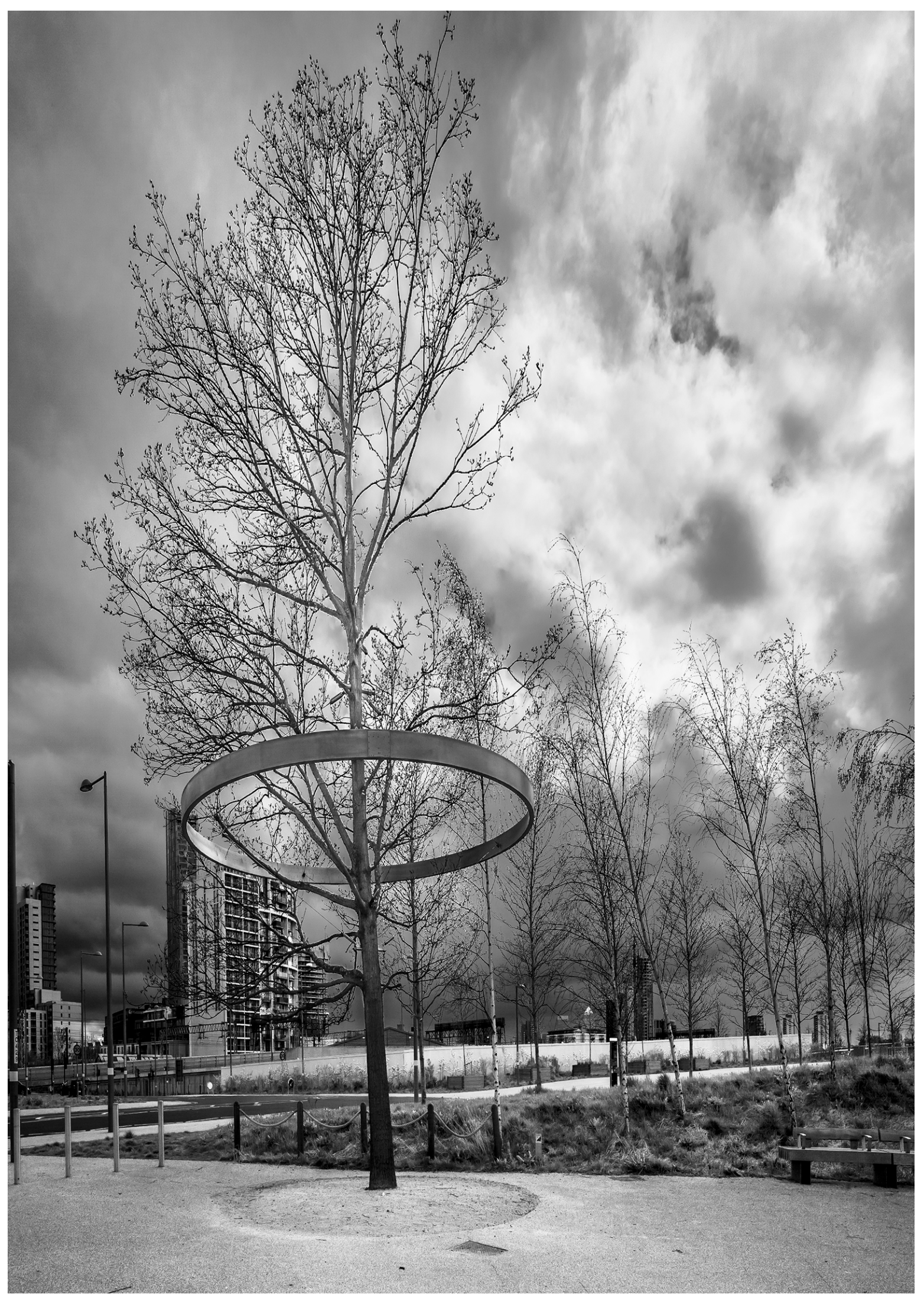

\section{PARTE III: Reflexiones finales}

¿Por qué es importante crear una visión de ciudad que incluya la cultura como eje estratégico?

Las últimas décadas de enfoques ad hoc han determinado que el proceso y el estado espacial resultante del proceso de urbanización, en muchas de las ciudades latinoamericanas, evidencia una organización del espacio que coincide con el fenómeno de la fragmentación urbana en el que los bordes impermeables y los enclaves tienen un papel central (Kozak, 2008, p. 256). Este desarrollo hacia una ciudad de islas, como lo define Janoshka, "resulta tanto del asentamiento insular de estructuras y funciones desde el momento de su construcción, como también del posterior aislamiento de espacios urbanos preexistentes mediante la construcción de rejas o muros" (2006, p. 86). Este estado de desunión y separación, el cual a menudo se combina con divisiones socioeconómicas o étnicas, lleva a cabo un acceso diferenciado y una inequidad de los recursos sociales.

En base a evidencia mostrada en casos revisados por la UNESCO (2017), las estrategias basadas en la cultura han ayudado a desarrollar las economías locales a abordar desigualdades. Adicionalmente, así como lo reconoce el Grupo de Trabajo Mundial de Gobiernos Locales y Regionales en el 2014, la cultura es un enfoque holístico e 
integrado para hacer frente a la actual crisis urbana, en un contexto en el que "la pobreza no solo es una cuestión de condiciones materiales e ingresos, sino también de carencia de capacidades y oportunidades, incluidos en términos culturales" (UNESCO, 2017, p. 205). En consecuencia, se deben de impulsar modelos de desarrollo que busquen enfrentar de manera integral e inclusiva la crisis urbana actual; así como lo definen Duxbury et. al (UNESCO, 2017, p. 205):

Además de disminuir la vulnerabilidad y la huella medioambiental, este nuevo modelo debe "rehumanizar" los entornos urbanos, tanto en términos de escala como en reforzar un sentimiento de pertenencia. Además, debe incrementar la cohesión social, contrarrestar la segregación (social y espacial) y la distribución desequilibrada de la riqueza y pretende la distribución y el acceso igualitario a los recursos urbanos y una mayor integración y conexión entre los residentes. Dentro de este contexto, debe reconocer que las culturas son dinámicas, intrínsecamente diversas y multifacetarias, que incorporan una gama de expresiones y valores encarnados en el patrimonio material e inmaterial, artes contemporáneas, actividades colectivas e individuales y características particulares dan forma a "modos de vida" distintos.

Con respecto a lo antes planteado, se hace evidente la oportunidad de impulsar un cambio de paradigma hacia una visión que promueva prácticas basadas en la comunidad y una dirección estratégica que se base en la cultura, la creatividad y el patrimonio, en forma de una política explícita o un documento de planificación que potencien ciudades diversas, inclusivas y prósperas. Como aportes, a continuación, se determinan tres elementos claves para su aproximación:

En primera instancia, es importante determinar que el desarrollo de una visión centrada en las personas denota la participación inclusiva de toda la ciudadanía, por ello requiere que todas las personas -de todos los grupos etarios- puedan acceder y contribuir a la vida cultural en una base de igualdad. Consecuentemente, como lo explica Yang (UNESCO, 2017, p. 136):

Esta colaboración necesitaría impulsar el sentido de solidaridad de las personas y la ciudadanía potenciando una conciencia de lugar y un sentimiento de pertenencia a la ciudad que puede apoyar a través del patrimonio y de las actividades que valoran la diversidad cultural de los habitantes de la ciudad. La vinculación de esto con los espacios públicos ha demostrado que promueve la interacción social positiva entre las personas y desarrolla la base económica de la ciudad..

En segunda instancia, el desarrollo de la visión debe de establecerse en un marco compuesto por una clara voluntad política, competencia técnica y recursos financieros. En tal sentido, el papel de las autoridades locales es fundamental para crear y apoyar espacios de acción y diálogo, planificar, diseñar, aplicar y supervisar políticas y programas, desarrollar infraestructuras y garantizar el reconocimiento de los valores del patrimonio, de la diversidad y de la creatividad, particularmente cuando estos se encuentran desatendidos o amenazados (UNESCO, 2017).

Y en tercera instancia, aunado al papel de los gobiernos en la creación de entornos propicios para guiar y estimular la creación de ciudades que se centren en las personas, se precisa definir dos niveles de intervención: un nivel en colaboración con el sector privado y la sociedad civil; y otro nivel que fomenta la participación directa de la ciudadanía a nivel de barrio. Es decir, la visión debe de desarrollarse conjuntamente; dar forma a la "cultura urbana" de la ciudad necesita consultas, sensibilización pública y debate según principios democráticos (UNESCO, 2017).

\section{Abordajes para la creación de políticas culturales}

Fukuda-Parr afirma que el multiculturalismo es mantener identidades múltiples para conseguir unidad en un contexto de diversidad, y es dentro de las ciudades que se maximizan las oportunidades de participación, interacción e intercambio transculturales y que "la política cultural es el único enfoque posible, porque la alternativa es la supresión de la cultura" (Agencia Española de Cooperación Internacional, 2004, p. 29). Bajo esta premisa, se aporta una propuesta de dos posibles abordajes para la creación de políticas culturales, con un tercer abordaje transversal, partiendo de la reflexión de 
la relación entre la cultura y ciudad, y que se basan en los derechos culturales de la ciudadanía.

El primer abordaje es la generación de políticas culturales que formen programas con una temporalidad específica. Esto implica que los programas tendrían un fuerte carácter de flexibilidad, el cual permita la variabilidad de los programas y proyectos en torno al crecimiento o evolución de las comunidades a la que están dirigidas. Asimismo, tendría una alta sensibilidad al reconocimiento de la diversidad cultural actual, concentrándose en modos creativos de promover redes entre las diversas identidades (étnicas, culturales, sociales, religiosas, entre otras), a través de los medios y recursos que van creándose a lo largo del tiempo.

El segundo abordaje, corresponde al aprovechamiento de la condición de aglomeración de las ciudades, lo cual implica, específicamente, traducir las políticas culturales a proyectos espaciales concretos. Un aspecto considera velar por la forma del espacio urbano, capitalizando las oportunidades que un buen diseño puede proveer para la convivencia entre culturas. Una segunda consideración es pautar criterios de diseño inclusivo, como una herramienta para que los gobiernos locales puedan adoptar efectivamente las políticas culturales en el territorio.

El tercer abordaje, partiendo del mismo aprovechamiento de la condición de aglomeración de las ciudades mencionado anteriormente, se centra en la necesidad de crear políticas culturales que se basan en procesos inclusivos de acceso, representación y participación de toda la ciudadanía en la cultura. Así como lo define la UNESCO (2017) las nuevas políticas culturales deben estar centradas en las personas y no en los sectores; se debe unir a todos los actores urbanos para que trabajen hacia la puesta en práctica de un nuevo modelo de desarrollo urbano sostenible que integre explícitamente dentro de sí la cultura. Este abordaje es de carácter transversal y articula la visión para el desarrollo urbano sostenible que incluye como temas la inclusión de los derechos humanos y la erradicación de todas las formas de discriminación en las ciudades. El diálogo con la ciudadanía en el ámbito local ha demostrado ser un mecanismo eficaz para crear políticas responsables basadas en las necesidades, y prestar servicios que empoderen a todos los miembros de la sociedad, ampliando sus habilidades de liderar el presente y decidir sobre el futuro.

Cabe destacar que el propósito de estos abordajes es continuar reivindicando los derechos culturales, con programas y proyectos que se ajusten a las particularidades de cada contexto cultural o territorial. Adicionalmente y para cerrar este escrito, se señala que, aunque no se mencionó anteriormente, se reconoce la necesidad de avanzar en estructuras innovadoras para los procesos de toma de decisiones, visualizando todos los roles (derechos y deberes) de cada actor involucrado en la creación o implementación de las políticas culturales en el mundo. Boldrini (2012) ha mantenido que la participación se trata de un ejercicio activo y sostenido en el tiempo mediante ámbitos y prácticas apropiadas y transformadoras, a través de las cuales se construyen vínculos interpersonales capaces de mantener el predominio de la integración comunitaria sobre la fragmentación. Esto requiere una inversión específica en la creación y fortalecimiento de capacidades, con una estrategia clara de intercambio de conocimientos, para que todos los actores urbanos estén mejor equipados para ser eficaces defensores de la cultura como una dimensión del desarrollo urbano. Con esta estructura, se visualiza una posible conservación de la pluralidad de culturas y patrimonio cultural mediante políticas y estrategias urbanas más informadas, inteligentes y sensibles con la cultura, capaces de guiar la construcción de ciudades inclusivas.

\section{Referencias bibliográfícas}

AgenciaEspañola de Cooperación Internacional.(2004). Derechos Culturalesy Desarrollo Humano. Publicación de textos del diálogo del Fórum Universal de las Culturas de Barcelona 2004. Tomado del Catálogo general de publicaciones oficiales: http:// publicaciones.administracion.es: http://publicaciones.administracion.es 
Asociación de Gestores y Técnicos Culturales. (sin fecha). Economía de la cultura. Ministerio de Cultura, Productor. Tomado de http://www.agetec.org/ageteca/ economia.htm http://www.agetec.org/ageteca/economia.htm

Banco Mundial (2019). Panorama general del desarrollo urbano. Tomado de: https:/l www.bancomundial.org/es/topic/urbandevelopment/overview

Boldrini, P. (2012). Producción participativa del hábitat popular en el área metropolitana de Tucumán. Tesis para optar al grado de Doctor de la Facultad de Filosofía y Letras. Universidad Nacional de Tucumán: Argentina.

Borja, J., y Castells, M. (1997). Local y global: la gestión de las ciudades en la era de la información. Madrid: Taurus.

Borja, J. y Muxí, Z. (2003). El espacio público, ciudad y ciudadanía. Barcelona: Electa.

Burden, A. (2014). How public spaces make cities work [Archivo de video]. Tomado de https://www.ted.com/talks/amanda_burden_how_public_spaces_make_cities_ work https://www.ted.com/talks/amanda_burden_how_public_spaces_make_ cities_work

Burgess, R. (2005). Technological determinism and urban fragmentation: a critical analysis. 9th International Conference of the ALFA-IBIS Network on Urban Peripheries, pp. 127-137. Pontificia Universidad Católica de Chile, Santiago de Chile, $11-13$ de julio.

Buendía, A., y Pino, J. C. (2011). Ciudad y diversidad cultural. Una aproximación desde la comunicación. Revista de Ciencias Sociales (RCS), XVII (1), 22-31.

Carrión, F. (2010). Ciudad, memoria y proyecto. Vol. V. OLACCHI: México. Tomado de: https://biblio.flacsoandes.edu.ec/libros/125313-opac

Carrión, F. (2016). El espacio público es una relación, no un espacio. En P. Ramírez (Ed.), La reinvención del espacio público en la ciudad fragmentada (pp. 13-47). Universidad Nacional Autónoma de México, México: Instituto de Investigaciones Sociales.

Castells, M. (1999). La era de la información. Vol. 1: La sociedad real. Barcelona: Editorial Siglo XXI.

Cervantes, C. (2005). Derechos culturales y desarrollo humano: implicaciones para el desarrollo de políticas culturales. Ponencia en III Encuentro Internacional de Promotores y Gestores Culturales "Desarrollo cultural: del pluralismo a la interculturalidad" Consejo Nacional para la Cultura y las Artes de México/UNESCO/ Secretaría de Cultura del Estado de Jalisco. Guadalajara, México.

Declaración de Friburgo. (2007). Tomado de: http://www.culturalrights.net/descargas/ drets_culturals239.pdf

Declaración sobre los Derechos de los Pueblos Indígenas de Naciones Unidas. (2007). Tomado de: http://www.un.org/esa/socdev/unpfii/documents/DRIPS_en.pdf" http://www.un.org/esa/socdev/unpfii/documents/DRIPS_en.pdf

Díez, M. (2015, 20 de enero). La cultura como motor de desarrollo. El País. Tomado de: https://elpais.com/elpais/2015/01/19/opinion/1421682870_125666.html" https:// elpais.com/elpais/2015/01/19/opinion/1421682870_125666.html

García, N. (2012). Culturas híbridas. Estrategias para entrar y salir de la modernidad. Barcelona: De Bolsillo.

García-Domenech, S. (2014). Percepción social y estética del espacio público urbano en la sociedad contemporánea. Arte, Individuo y Sociedad, 26 (2), pp. 301-316. ISSN: $1131-5598$

Gehl, J. (2014). Ciudades para la gente. Ciudad Autónoma de Buenos Aires: Infinito.

Jacobs, J. (1961). The Death and Life of Great American Cities. Nueva York: Random House.

Janoschka, M. (2006). El modelo de ciudad latinoamericana. Privatización y fragmentación del espacio urbano de Buenos Aires: el caso Nordelta. Buenos Aires a la deriva. Transformaciones urbanas recientes, pp. 96-131. 
Kozak, D. (2008). Urban Fragmentation in Buenos Aires: The Case of Abasto [tesis de doctorado, Oxford]. Oxford Brookes University.

Kozak, D. (2018). Revisitando el debate sobre la Fragmentación Urbana. Revista Iberoamericana de Urbanismo, $\mathrm{n}^{0}$ 14, pp. 15-22. Tomado de: https:// upcommons.upc.edu/bitstream/handle/2117/130389/14_03_RIURB_kosak. pdf? sequence $=1$ \&isAllowed=y

Mejías C. y Henríquez, P. (2012). La Ciudadanía como Co-Construcción de Espacios de Participación en lo Público. Sociologias, Porto Alegre, año 14, No. 31, set./ dez., pp. 192-213

Norberg-Schulz, C. (1975). Existencia, espacio y arquitectura. Barcelona: Blume.

Pascual, J. (2015). Relatos y actores sobre la cultura en la mundialización [Material de clase]. Derechos culturales y mundialización. Módulo 1. Universitat Oberta de Catalunya.

PNUD. (2004). Informe sobre Desarrollo Humano 2004. La libertad cultural en el mundo diverso de hoy. Programa de las Naciones Unidas para el Desarrollo. Ediciones Mundi-Prensa.

Seijo, L. (2014). La ciudad como escenario: Prácticas artísticas en el espacio público. Reflexión Académica en Diseño y Comunicación. Año XVI. Vol. 24. (2015). pp. 208-211. ISSN 1668-1673

Scott, A. (2001). Capitalism, cities, and the production of symbolic forms. (R. G. Society, Ed.) Transactions of the Institute of British Geographers, 13, 11-23.

UNESCO (2017). Cultura: Futuro Urbano. Informe Mundial sobre la cultura para el desarrollo urbano sostenible. París: Organización de las Naciones Unidas para la Educación, la Ciencia y la Cultura.

UNESCO (2020). Textos fundamentales. Edición de 2020. Organización de las Naciones Unidas para la Educación, la Ciencia y la Cultura: París. Tomado de: https:// unesdoc.unesco.org/ark:/48223/pf0000372956_spa/PDF/372956spa.pdf.multi. page $=7$

Vargas, J. M. (2003). Universidad Complutense de Madrid (UCM). Tomado de: Espéculo: Reseñas, críticas y novedades: https://webs.ucm.es/info/especulo/numero23/ dicciona.html" https://webs.ucm.es/info/especulo/numero23/dicciona.html

Wessel, T. (2009). Does diversity in urban space enhance intergroup contact and tolerance? Geografiska Annaler: Series B, Human Geography 91 (1), pp. 5-17. 